\title{
Physical analysis of a processive molecular motor: The conventional kinesin
}

\author{
A. Ciudad, ${ }^{1, *}$ A. M. Lacasta, ${ }^{2}$ and J. M. Sancho ${ }^{1}$ \\ ${ }^{1}$ Departament d'Estructura i Constituents de la Matèria, Facultat de Física, Universitat de Barcelona, Diagonal 647, E-08028 \\ Barcelona, Spain \\ ${ }^{2}$ Departament de Física Aplicada, Universitat Politècnica de Catalunya, Av. Dr. Marañón 44, E-08028 Barcelona, Spain
}

(Received 22 April 2005; published 29 September 2005)

\begin{abstract}
We present a model that allows for the derivation of the experimentally accesible observables: spatial steps, mean velocity, stall force, useful power, efficiency and randomness, etc. as a function of the [adenosine triphosphate] concentration and an external load $F$. The model presents a minimum of adjustable parameters and the theoretical predictions compare well with the available experimental results.
\end{abstract}

DOI: 10.1103/PhysRevE.72.031918

PACS number(s): 87.16.Nn, 05.10.-a

\section{INTRODUCTION}

The highly specialized and localized activity inside an eukaryotic cell needs directional (not random as diffusion) transport of intracellular structures such as vesicles or mitochondria. This job is done by some wide families of motor proteins acting as nanometrical devices which convert chemical energy into mechanical work. They operate in a relative strong thermally fluctuating medium but with the supporting help of a rigid and periodic structure that makes possible and defines the direction of the active transport. The source of chemical energy is provided by the hydrolysis of the adenosine triphosphate (ATP).

Molecular motors are receiving a lot of interest not only from a biomolecular point of view but from a physical one. We will focus our attention on a particular motor, the conventional kinesin. The reason is that recent single molecule experiments with this protein have provided very useful data introducing unexpected features that our model tries to explain [1]. We expect that a similar approach can be derived for other molecular motors, incorporating their more specific ingredients [2].

A single or isolated molecular motor operates cyclically by presenting conformational changes (mechanical motion) fueled by chemical reactions, and afterwards reseting spontaneously to the initial configuration in order to restart another cycle [3]. We expect that mechanical motion steps have to be coupled to the chemical reaction steps that generate the energy for this mechanical motion [4].

It is commonly accepted that Brownian motion or thermal fluctuations are essential ingredients in this cycle [5]. Actually what we do know is that thermal fluctuations are necessary for crossing potential barriers and for diffusion. Moreover they are also responsible for the dissipation and loss of energy through fluctuation-dissipation relations. From the physical point of view all of these engines have to share basic and common observables such as spatial steps, mean velocity, useful power, efficiency, and randomness $[3,4]$. The role of these minimum sets of ingredients has to be clear in any proposed model independently of its complication. Such

\footnotetext{
*Corresponding author. Email-address: aciudad@ecm.ub.es
}

simplified theories or minimum models also have to cover in a clear way the coupling between chemical energy consumption and external useful power. This is so because all these aspects are experimentally accessible to some extent.

In our model, we pay special attention to the energetics of the processes involved in the kinesin cycle and the way they couple to mechanical movement, instead of taking much care of intermediate steps. Using a reduced number of parameters, but all of them with physical or chemical meaning, we are able to reproduce the observed quantities like the mean velocity, the randomness, or the stall force, in good agreement with the data obtained in experiments. The control variables are the external load, $F$, controlled in experiments by using force clamps and the ATP concentration [ATP].

In Sec. II we present the analytical study of an ideal motor that admits analytical treatment giving rise to the qualitative behavior and a discussion of some important points. In Sec. III we proceed with a more realistic kinesin model, taking into account biochemical and physical inputs. Different hypothesis are discussed in detail. In Sec. IV theoretical results are presented and compared with experimental data. Finally, in Sec. $\mathrm{V}$ we end with a set of comments and discussions.

\section{THE IDEAL MOTOR}

Here we will present a discussion on the most relevant aspects of a molecular motor using a very simple or ideal model. Molecular motors operate over a track or other type of repetitive structure made of proteins. The motor-track interaction is probably the most important interaction in the system, the others being less relevant and which can be incorporated into the thermal bath or other degrees of freedom. So we will assume that the most relevant variable is the position of the motor along the symmetry axis of the filament. The chemical variables and the track are incorporated through an effective free energy using $\Delta G_{u}$ in a step $l_{o}$.

We will propose the following Langevin equation that allows a simple analytical approach and can be useful to discuss some relevant aspects of the problem and to obtain preliminary estimated values for some parameters. The equation of motion is 


$$
\lambda \dot{x}=\frac{\Delta G_{u}}{l_{0}}-F+\xi(t),
$$

where $\xi(t)$ is the thermal force, responsible for the strong fluctuations, which is a Gaussian white noise with a correlation given by

$$
\left\langle\xi(t) \xi\left(t^{\prime}\right)\right\rangle=2 \lambda k_{B} T \delta\left(t-t^{\prime}\right),
$$

according a fluctuation-dissipation relation. $F$ is the external load and $\lambda$ is the friction parameter. Here the motor moves under a chemical constant force $\Delta G_{u} / l_{0}$ which mimics exactly one ATP consumed for $l_{0}$. This simple modelization does not incorporate the activation energy of the ATP hydrolysis. This important ingredient is addressed in the next section.

Our interest is to find a prediction for the mean velocity $\langle v\rangle$ and for the randomness parameter defined as

$$
r=\lim _{t \rightarrow \infty} \frac{\left\langle\Delta x(t)^{2}\right\rangle}{l_{0}\langle v(t)\rangle t}=\frac{2 D}{\langle v\rangle l_{0}},
$$

where $D$ is the diffusion coefficient. These two observables have to be obtained as a function of the experimental external control parameters: the ATP concentration and the external load $F$.

As this model allows a quick solution we get

$$
\begin{gathered}
\langle v\rangle=\frac{1}{\lambda}\left(\frac{\Delta G_{u}}{l_{0}}-F\right), \\
\left\langle\Delta x(t)^{2}\right\rangle \sim \frac{2 k_{B} T}{\lambda} t=2 D t .
\end{gathered}
$$

These predictions disagree with experimental data but their careful analysis will allow us to introduce the observables we want to study, to discuss important aspects, and to get relevant information to make more accurate improvements in the model.

From Eq. (4), one can obtain that the stall force $(\langle v\rangle=0)$ is $F_{S}=\Delta G_{u} / l_{0}$. As from the experimental data of Ref. [1] we observe that $F_{s}$ increases very slowly with the ATP concentration, we expect the same dependence for $\Delta G_{u}$. Nevertheless the experimental velocities do depend very much on ATP concentration so we need a new term in the model but maintain the prediction for $F_{S}$.

Equations (3)-(5) give a randomness,

$$
r=\frac{2 k_{B} T}{\Delta G_{u}-F l_{0}} .
$$

This prediction is qualitatively similar to experimental data of Ref. [1] with a divergence of $r$ at the stall force. Nevertheless the predicted values are very small. This is due because all the free energy $\Delta G_{u}$ is taken directly without any activation energy barrier. An important point to stress is that Eq. (6) predicts that $r$ does not depend on the friction parameter.

Let us analyze a set of quantities we are interested in. The mean useful power produced by the motor against the external load $F$ is

$$
W_{F}=F\langle v\rangle .
$$

Using Eq. (4) we get

$$
W_{F}=\frac{F}{\lambda}\left(\frac{\Delta G_{u}}{l_{0}}-F\right),
$$

which presents the standard parabolic form of any kind of motor.

The mean power of the chemical energy consumption is,

$$
W_{G}=\frac{\Delta G_{u}\langle v\rangle}{l_{0}},
$$

which directly follows from the assumption that one ATP molecule, $\Delta G_{u}$, is consumed per track step $l_{0}$. One can use the benchmark experiments using optical tweezers to estimate the efficiency against a conservative external force, which is

$$
\varepsilon=\frac{F l_{0}}{\Delta G_{u}} .
$$

Although the maximum of efficiency is given for $F=F_{S}$ or $\langle v\rangle \sim 0$, we get that the maximum power, using Eq. (8), appears for $F=F_{S} / 2$, with a very optimistic efficiency of $50 \%$. However, working in vivo conditions the chemical input of energy is dissipated by drag forces or other degrees of freedom.

An estimation of the value taken by the friction parameter $\lambda$ needs a careful analysis of the experimental data and the model predictions. Let us take now $F=0$ (free motion) and using that the experimental mean velocity under saturated concentration of ATP is $\sim 800 \mathrm{~nm} / \mathrm{s}$ [1], we can get an upper value prediction for the friction parameter $\lambda=\Delta G /\langle v\rangle_{\max } l_{0}$ $\sim 10^{-2} \mathrm{pN} \mathrm{s} / \mathrm{nm}$. This is a quite surprising result. In the literature we found $\lambda \sim 10^{-7} \mathrm{pN} \mathrm{s} / \mathrm{nm}$ in Refs. [6-8], or a similar value as here in [9], for a similar modelization. A physical discussion is worth including here. From an estimation of the Stokes friction $\lambda=6 \pi \eta R$, using the viscosity of the water $\eta=10^{-1}$ Pas and the radius of the silica bead $R=0.5 \mu \mathrm{m}$ [1], one can obtain a estimated value of $\lambda \sim 10^{-5} \mathrm{pN} \mathrm{s} / \mathrm{nm}$, which is also very far from the value we have obtained. Our interpretation of this fact is that, as it occurs in other stepping engines, the mean velocity is essentially given by the dwell time between steps. Then, because these times are controlled by the ATP concentration, the value of the drag force in this case is not physical but chemical. As we will see below a better estimation of this parameter will reduce this value but not too much. As a conclusion we expect a larger effective friction parameter that the one obtained from the viscosity of the water. All this information will be used to propose a more realistic model with a minimum of fitting parameters which will give predictions with a better experimental agreement.

\section{THE MODEL}

Before we present the model let us analyze the main biochemical facts that have, in our opinion, to be incorporated in any molecular model. 


\section{A. Biochemical inputs}

From experiments $[1,10]$ it can be seen that kinesin moves with discrete steps of order $8.2 \mathrm{~nm}$ ([20]). We will call this distance $l_{0}$ and it will be the first fixed constant of our model.

The introduction of the chemical energy input needs a more careful analysis than in the ideal model. It is a commonly accepted biochemical hypothesis, sustained by an interpretation of well-prepared experiments [11], that the kinesin hydrolyzes one ATP to perform one step. Other motors, like Myosin V, operate in the same way but with a different $l_{0} \sim 37 \mathrm{~nm}$ [12]. This tight coupling between one unit of free energy consumed and one elementary step implies that a precise value of the free energy available in the ATP hydrolysis, $\Delta G_{u}$, is a crucial quantity. In textbooks [2] one can read that the free energy $\Delta G$ assigned to ATP hydrolysis reaction,

$$
\mathrm{ATP} \rightleftharpoons \mathrm{ADP}+\mathrm{P}_{i}
$$

is given by

$$
\Delta G=\Delta G^{0}+k_{B} T \ln \left(\frac{[\mathrm{ATP}]}{[\mathrm{ADP}]\left[\mathrm{P}_{i}\right]}\right),
$$

where $\Delta G^{0}=55 \mathrm{pN} \mathrm{nm}$ is the equilibrium value and $\mathrm{P}_{i}$ stands for inorganic phosphate. Although the standard free energy $\Delta G^{0}$ depends on $p \mathrm{H}$, ionic strength, and other factors, the standard conditions of the experiments should not move this value. However, in order to calculate the total free energy, ADP and $\mathrm{P}_{i}$ concentrations are relevant. Commonly accepted values for these concentrations are of order $10 \mathrm{nM}$ [13], and they do not change significatively for different solutions of $[\mathrm{ATP}]$. Then we will have finally the following expression:

$$
\Delta G=\left(55+4.1 \ln \left(10^{4}[\mathrm{ATP}]\right)\right) \mathrm{pN} \mathrm{nm},
$$

where we have taken $k_{B} T \sim 4.1 \mathrm{pN} \mathrm{nm}$, corresponding to a temperature of $25^{\circ} \mathrm{C}$.

Another important question is the percentage of this free energy that the motor is able to use. Although the mechanism of using this free energy remains under scrutiny, there are experimental evidences, see below, that indicate that the hydrolysis of one ATP is not used exclusively to advance a forward step. In Ref. [2] it is remarked that, according to the observed stall forces, only half of the thermodynamic free energy is avaliable to this movement. According to this conjecture we will assume that the used chemical energy is

$$
\Delta G_{u}=\frac{\Delta G}{2}
$$

\section{B. Physical inputs}

We are going to consider only axial movements along the track and we only interested in following the center of the mass of the motor. An overdamped Langevin dynamics is the most simple way to express an equation for its position. The equation reads

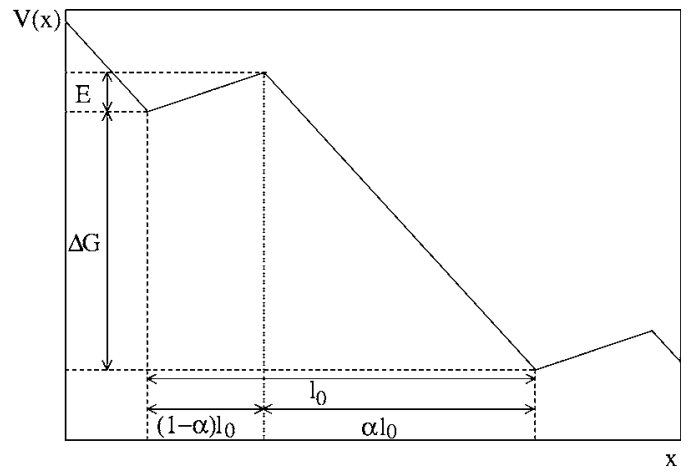

FIG. 1. Scheme of the effective potential with all the parameters and in an arbitrary scale.

$$
\lambda \dot{x}=-V^{\prime}(x)-F+\xi(t)
$$

where $\xi(t)$ is the same thermal force than in the ideal model.

$V(x)$ is the potential that the motor is subjected in, and it incorporates all the necessary biochemical ingredients of this problem. In Ref. [4] a piecewise effective potential is proposed. Now we will develop this hypothesis. The kinesin advances in a periodic track of length $l_{0}$ in a periodic effective potential given by

$V(x)$

$$
=\left\{\begin{array}{l}
\frac{E}{l_{0}(1-\alpha)} x, 0<x \leqslant l_{0}(1-\alpha) \\
-\frac{\left(\Delta G_{u}+E\right)}{\alpha l_{0}} x+\frac{E+(1-\alpha) \Delta G_{u}}{\alpha}, l_{0}(1-\alpha)<x \leqslant l_{0},
\end{array}\right.
$$

which is plotted in Fig. 1.

Here we can see that the free energy $\Delta G_{u}$ consumption is tightly coupled with the step $l_{0}$. The only adjustable parameters of the model are the activation barrier $E$ which deserves a detailed study (next section) and the parameter $\alpha$ which measures the relative spatial length occupied by the chemical processes. (This parameter appears also in Ref. [9].) Concretely, the asymmetric parts of the resulting ratchet potential correspond to the capture and hydrolysis of an ATP (small part) and to the advancing step of one of the heads (large part). We will see later how only a very small fraction of the step corresponds to the capture and hydrolysis, leading to a nonspatiality of the chemical reaction coordinate. The parameters $\alpha$ and $\lambda$ are estimated from a best fitting of the experimental data of Ref. [1].

\section{The activation energy $E$}

As we have already mentioned, there is an energy barrier that makes necessary the presence of thermal fluctuations. This barrier is also responsible for the values of the randomness. For a molecule of ATP, the process of hydrolysis is just to break the covalent pyrophosphate bond, and it means that an amount of energy $E$, given by thermal fluctuations, is needed. However, while in the cytoplasm $E$ is so high that 
the reaction hardly happens spontaneously, in the kinesin's catalytic domain $E$ will be reduced considerably because of the enzymatic properties of the protein. On the other hand, at high [ATP] the kinetics will saturate as expected in any Mchaelian enzymatic process. Even in these conditions a little barrier $E_{0}$ will remain. We will estimate the [ATP] influence from experimental data. The kinetics of a barrier crossing is described by Arrhenius' (experimental) or Kramers (theoretical) law, and it predicts an exponential decay for the reaction rate. We will assume here that for this enzymatic reaction we have the form

$$
k=k_{0} e^{-E / k_{B} T},
$$

where the limiting constant $k_{0}$ depends on the shape of the potential and on the friction. Moreover, the effect of ATP concentration is taken into account by assuming a MichaelisMenten saturating expression,

$$
k=k_{\max } \frac{[\mathrm{ATP}]}{K_{M}+[\mathrm{ATP}]},
$$

where $k_{\max }$ is the saturating reaction rate at very high [ATP] which introduces the parameter $E_{0}$ as

$$
k_{\max }=k_{0} e^{-E_{0} / k_{B} T} .
$$

Our hypothesis is to consider that the whole process has a single effective barrier that, in consequence, has to depend on [ATP]. Then, joining Eqs. (17)-(19) we get an expression for $E$ that depends on [ATP] in the following way:

$$
E=E_{0}+k_{B} T \ln \left(1+\frac{K_{M}}{[\mathrm{ATP}]}\right)
$$

which has only two free parameters, $E_{0}$ and $K_{M}$. It is worth commenting that this approach is the result of a two step process, each one having a characteristic time scale. The first step represents the capture of an ATP molecule, and the associate time scale will be proportional to $1 /[\mathrm{ATP}]$. The second step is the hydrolysis of the nucleotide, and its time scale will be Kramers-like, that is, proportional to $e^{E_{0} / k_{B} T}$.

Using now experimental data of Ref. [1] we have found that the best values are $E_{0}=1 \mathrm{pN} \mathrm{nm} \sim 0.25 k_{B} T$ (the remaining barrier in saturating conditions) and $K_{M}=1020 \mu \mathrm{M}$ (the concentration when the kinesin is $50 \%$ saturated). It is important to notice that at high ATP concentrations, the height of the effective barrier is not important, according to the ideal situation of the previous section. The rest of parameters, $\alpha$ and $\lambda$, are also estimated. We have found the values $\alpha=0.97$ and $\lambda=0.008 \mathrm{pN} \mathrm{s} / \mathrm{nm}$, which can be compared with the values used in Ref. [9]: $\alpha=0.73$ and $\lambda=0.01 \mathrm{pN} \mathrm{s} / \mathrm{nm}$. The values of the drag force are similar and correspond to a high and nonhydrodynamical friction. However, $\alpha$ is bigger in our case, although there is no surprise in this result: in the model of Ref. [9] there are no changes of the potential in the short segments, while in our case, this short segment has a pronunciated upward slope. This fact could explain the discrepancy.

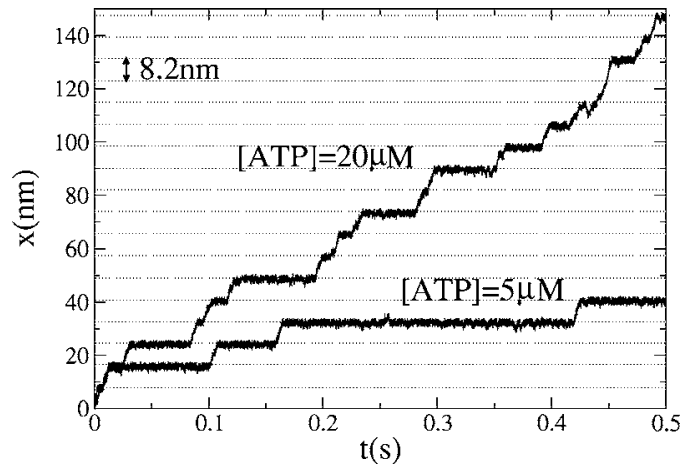

FIG. 2. Kinesin trajectories for $[\mathrm{ATP}]=5$ and $[\mathrm{ATP}]$ $=2000 \mu \mathrm{M}$ from numerical simulation of model (15).

\section{THEORETICAL PREDICTIONS VERSUS EXPERIMENTAL DATA}

Our model (15) allows some analytical calculations because the variable position is a Markovian process which follows a standard Fokker-Planck equation. The mean velocity $\langle v\rangle$ and the diffusion coefficient can be expressed in quadratures [14],

$$
\begin{gathered}
\langle v\rangle=\frac{l_{0} k_{B} T}{\lambda} \frac{\left(1-e^{\left(F l_{0}-\Delta G_{u}\right) / k_{B} T}\right)}{\int_{0}^{l_{0}} d x I_{+}(x)}, \\
D=\frac{k_{B} T l_{0}^{2}}{\lambda} \frac{\int_{0}^{l_{0}} d x I_{+}^{2}(x) I_{-}(x)}{\left[\int_{0}^{l_{0}} d x I_{+}(x)\right]^{3},}
\end{gathered}
$$

where

$$
I_{ \pm}(x)= \pm \frac{\lambda e^{\mp U(x) / k_{B} T}}{k_{B} T} \int_{x}^{x \pm l_{0}} d y e^{ \pm U(y) / k_{B} T}
$$

and

$$
U(x)=V(x)+F x .
$$

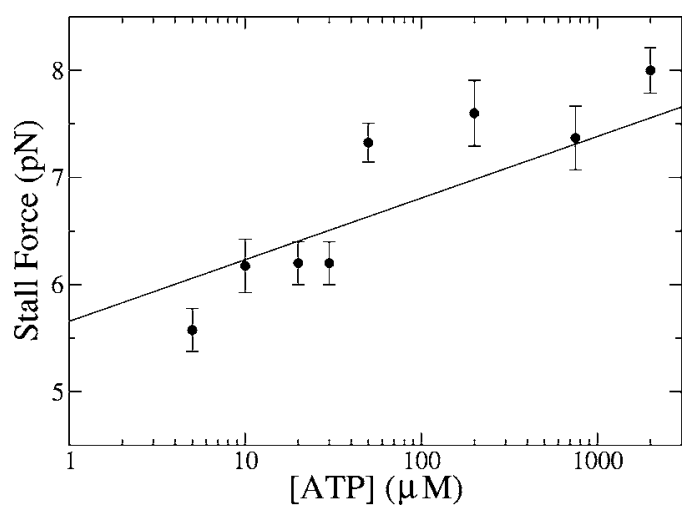

FIG. 3. Stall Force (pN) (using a position clamp) versus [ATP] concentration. The straight line is the analytical prediction (25) and dots are the experimental data of Ref. [1]. 


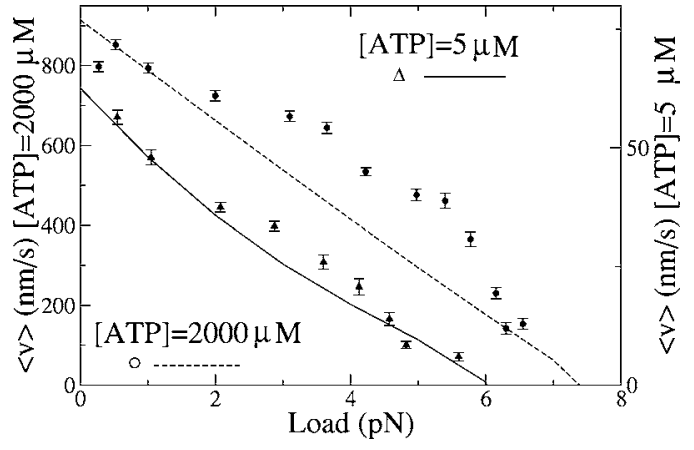

FIG. 4. Mean velocity $(\mathrm{nm} / \mathrm{s})$ vs $F(\mathrm{pN})$ for two [ATP] concentrations. Points are from experimental data of Ref. [1]. Lines are theoretical predictions.

With these expressions the relevant quantities can be evaluated numerically and compared now with the experimental data. First we will present, in Fig. 2, two representative trajectories of the kinesin motion as they come from numerical simulations of Eq. (15). These trajectories present the typical stepping movement of the kinesin which are more clear for low [ATP]. In this figure, we see how the motor advances faster for the higher ATP concentration case.

The first analytical prediction or our model is that the stall force $F_{S}$ has the same expression as in the ideal motor,

$$
F_{S}=\frac{\Delta G_{u}}{l_{0}}
$$

but now in terms of $\Delta G_{u}$ given by Eq. (13), which is plotted in Fig. 3.

The velocity versus load is plotted in Fig. 4. The agreement is quite good although we will comment on this point in our conclusions. In Fig. 5 we can see the mean velocity versus ATP concentrations for different values of the load.

Finally, the randomness is plotted in Figs. 6 and 7 in front of the load and [ATP], respectively. It is clear that, though the qualitative behavior is well-described, we underestimate the value of the randomness, especially at low [ATP].

At this point it is worth discussing the efficiency of the kinesin from experimental observations. One can take that

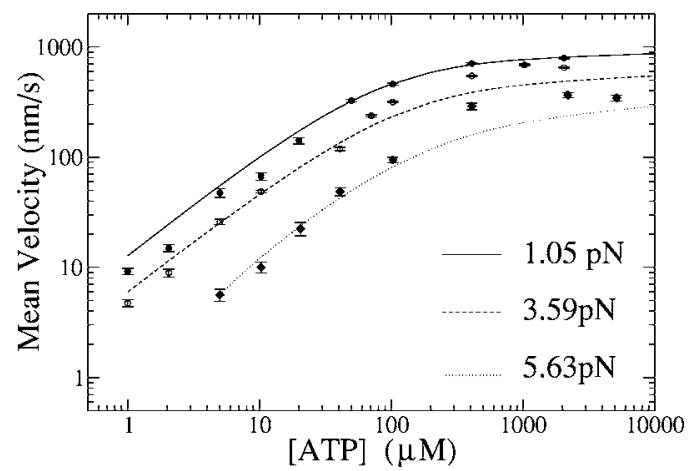

FIG. 5. Mean velocity vs [ATP] for different values of the load.

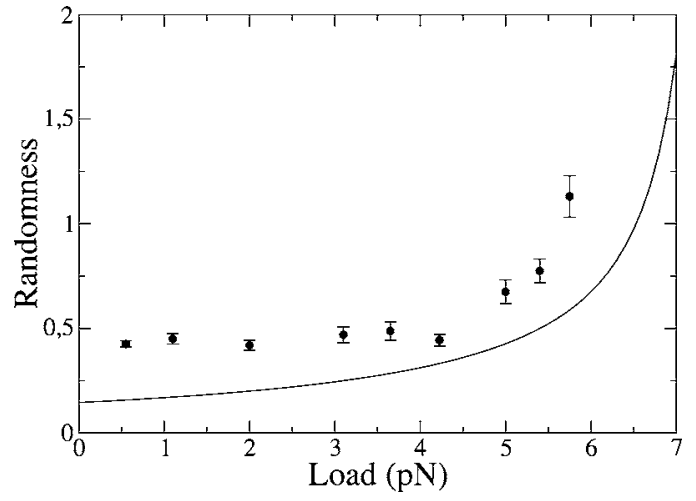

FIG. 6. Randomness vs $F$ for $[\mathrm{ATP}]=2 \mathrm{mM}$.

the [ATP] concentration inside a cell is of the order of $\mathrm{mM}$ [15-17]. If we plot the mean power versus load for the experimental points of Ref. [1] for [ATP] $=2 \mathrm{mM}$, we can see that the maximum power takes place at a value of $F$ $\sim 5 \mathrm{pN}$. Using Eq. (12) we get $\Delta G \sim 124 \mathrm{pN} \mathrm{nm}$ so the efficiency (10) is just $\epsilon=F l_{0} / \Delta G \sim 0.33$. We conjecture that this is the order of efficiency that one has to expect in any of the molecular motors as myosins [18], and never quite unrealistic values of the order of $100 \%$ [19].

\section{COMMENTS AND CONCLUSIONS}

We have presented a simple model of a molecular motor which is able to predict experimental observations for the conventional kinesin. The agreement, although qualitatively good, is not optimal. The experimental velocity-load curves have some discrepancies especially at high [ATP]. This is due to the simplicity of the model which skips second-order factors that probably are responsible of this disagreement.

The results for the randomness, although qualitatively correct, give systematically underestimated values with respect to the experiments. This discrepancy is also seen in other models like [15]. We believe that in order to improve this prediction, a more detailed description of the role of the external force in each of the chemical states is needed [20]. Concretely, the Michaelis constant should be considered load-dependent due to the coordinated cycles of the two

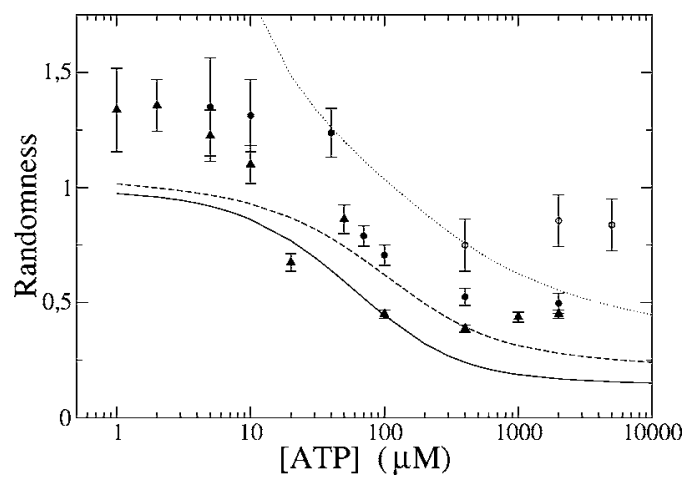

FIG. 7. Randomness vs [ATP]. The values of $F$ are the same as in Fig. 5. 
catalytic domains of the dimer. This is a key point for further improvements, although more refined experimental data would be more clarifying.

The model proposed here is a clear improvement of the more simple model presented in Ref. [9]. Our model incorporates the ATP concentration and the external load as independent variables. Moreover, the quality of our predictions compares favorably with respect to the modelizations of Ref. [15] which, for only some observables, obtain a better agreement with the experiments but pay the price of using more adjustable parameters. Furthermore, this model does not take a kinetic approach. Instead, it takes into account how the motor diffuses along a coordinate reaction. Then, we do not consider the velocity of the step to be infinite.

Thus our model provides a very good balance between mathematical simplicity, physico-biochemical hypothesis, and experimental results.

\section{ACKNOWLEDGMENTS}

This research was supported by the Ministerio de Educación y Ciencia (Spain) under Project No. BFM200307850-C03-01-02 and Grant No. BES-2004-3208. We thank G. P. Tsironis for his comments.
[1] K. Visscher, M. J. Schnitzer, and S. M. Block, Nature (London) 400, 184 (1999).

[2] J. Howard, Mechanics of Motor Proteins and the Cytoskeleton (Sinauer Associates, Sunderland, 2001).

[3] C. Bustamante, D. Keller, and G. Oster, Acc. Chem. Res. 34, 412 (2001).

[4] H. Wang and G. Oster, Appl. Phys. A: Mater. Sci. Process. 75, 315 (2002).

[5] G. Oster, Nature (London) 417, 25 (2002).

[6] D. Astumian, in Modern Challenges in Statistical Mechanics: Patterns, Noise, and the Interplay of Nonlinearity and Complexity, edited by V. M. Kenkre and K. Lindenberg, AIP Conf. Proc. No. 658 (AIP, Melville, New York, 2003).

[7] G. N. Stratopoulos, T. E. Dialynas, and G. P. Tsironis, Phys. Lett. A 252, 151 (1999).

[8] T. Shimokawa, S. Sato, A. Buonocore, and L. M. Ricciardi, BioSystems 71, 179 (1993).

[9] M. Bier, Phys. Rev. Lett. 91, 148104 (2003).
[10] C. M. Coppin et al., PNAS 94, 8539 (1997).

[11] M. J. Schnitzer and S. M. Block, Nature (London) 388, 386 (1997); W. Hua et al., ibid. 388, 390 (1997).

[12] M. Rief et al., PNAS 97, 9482 (2000).

[13] C. L. Asbury, A. N. Fehr, and S. M. Block, Science 2130, 302 (2003).

[14] B. Lindner and L. Schimansky-Geier, Phys. Rev. Lett. 89, 230602 (2002).

[15] M. E. Fisher and A. B. Kolomeisky, PNAS 98, 7748 (2001).

[16] E. Hirakawa, H. Higuchi, and Y. Y. Toyoshima, PNAS 97, 2533 (2000).

[17] F. M. Gribble et al., J. Biol. Chem. 275, 30046 (2000).

[18] Zhen-He He et al., Biophys. J. 79, 945 (2000).

[19] K. Kinosita, Jr. et al., Philos. Trans. R. Soc. London, Ser. B 355, 473 (2000).

[20] S. M. Block, C. L. Asbuty, J. W. Shaevitz, and M. J. Lang, PNAS 100, 2351 (2003). 